MANNHEIM RESEARCH INSTITUTE For THE ECONOMICS OF AGING

\title{
ZUR SINNHAFTIGKEIT DER \\ RIESTER-RENTE
}

\section{Axel Börsch-Supan}

Martin Gasche

๑) $\boldsymbol{m e a - M a n n h e i m ~ R e s e a r c h ~ I n s t i t u t e ~ f o r ~ t h e ~ E c o n o m i c s ~ o f ~ A g i n g ~}$

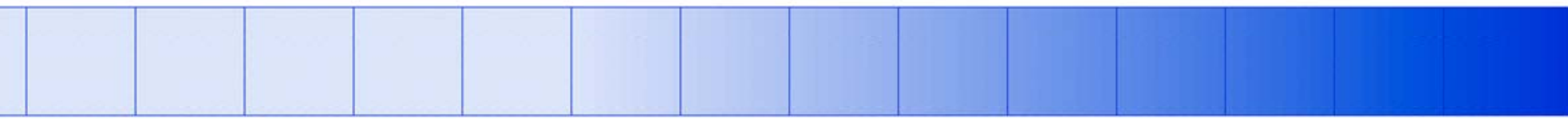

L13, 17_D-68131 Mannheim_Phone +49 621 181-2773/1862_Fax +49 621 181-1863_www.mea.uni-mannheim.de 


\title{
Zur Sinnhaftigkeit der Riester-Rente
}

\author{
Axel Börsch-Supan \\ Martin Gasche \\ Mannheimer Forschungsinstitut Ökonomie und Demographischer Wandel (MEA) \\ Universität Mannheim*
}

Januar 2010

\begin{abstract}
Zusammenfassung
Im Jahr 2001 wurde mit der Einführung der so genannten Riester-Rente ein Paradigmenwechsel in der Altersvorsorge eingeleitet. Durch die Finanzkrise 2008/09 ist die Frage, wie groß das Risiko der kapitalgedeckten Altersvorsorge ist, jedoch wieder aktuell geworden. Vor allem wird diskutiert, ob nicht die Höhe der krisenbedingten Vermögensverluste und deren Prozyklität daran zweifeln lässt, dass ein Mischsystem aus Kapitaldeckung und Umlage den Herausforderungen der demographischen Entwicklung am besten gewachsen ist.

Dieser Beitrag stellt klar, dass zu einer grundsätzlichen Neuordnung des 2001 eingeführten Mehrsäulenmodells der deutschen Altersvorsorge kein Anlass besteht. Der mittlere krisenbedingte Verlust des Altersvorsorgevermögens liegt bei etwa 2\%. Modellierungen der makroökonomischen Effekte mit kurzfristig orientierten Modellen ergeben in der Tat prozyklische Effekte, diese Modellansätze sind dem langfristigen Charakter der privaten Altersvorsorge jedoch völlig unangemessen.
\end{abstract}

JEL: G01, E27, G11, J26

Schlagwörter: private Altersvorsorge, Finanzkrise

\footnotetext{
* Das MEA ist ein Forschungsinstitut der Universität Mannheim, das sich zu etwa zwei Dritteln aus Drittmitteln der öffentlichen Forschungsförderung finanziert. Dafür sind wir dankbar. Wir danken ebenso dem Land BadenWürttemberg und dem Gesamtverband der Deutschen Versicherungswirtschaft für die Grundfinanzierung des MEA. Wir danken Dr. Anette Reil-Held für hilfreiche Kommentare.
} 


\section{Einleitung}

Im Jahr 2001 wurde in Form der so genannten Riester-Rente ein Paradigmenwechsel der Altersvorsorge eingeläutet. Zwar gab es schon des Längeren private betriebliche und individuelle Altersvorsorge, sie war jedoch das „Sahnehäubchen“ auf der den Lebensstandard sichernden gesetzlichen Rente. Seit den großen Reformen 2001 („Riester“) und 2004 („Nachhaltigkeit“) ist ein Anteil privater Vorsorge unumgänglich, um den während des Erwerbslebens genossenen Lebensstandard auch im Ruhestand zu sichern. Diese Reformen lassen sich als Teilübergang von einem Umlageverfahren zum Kapitaldeckungsverfahren interpretieren, wobei auch in Zukunft das Umlageverfahren für den Durchschnittsbürger bei weitem dominieren wird. Insgesamt ist dieser Übergang weitgehend akzeptiert worden, auch im unteren Einkommenssegment sind die Zuwachsraten bei der Riester-Rente groß. ${ }^{1}$

Durch die Finanzkrise 2008/09 ist die Frage, wie groß das Risiko der kapitalgedeckten Altersvorsorge ist, jedoch wieder aktuell geworden. Vor allem wird diskutiert, ob nicht die Höhe der krisenbedingten Vermögensverluste und deren prozyklische Wirkung daran zweifeln lassen, dass ein Mischsystem aus Kapitaldeckung und Umlage den Herausforderungen der demographischen Entwicklung am besten gewachsen ist.

Wir sehen keinen Anlass zu einer grundsätzlichen Neuordnung des von der Rot-GrünenKoalition eingeführten Mehrsäulenmodells der deutschen Altersorge. Der mittlere krisenbedingte Verlust des Altersvorsorgevermögens liegt in der gleichen Größenordnung wie der Verlust an gesetzlichen Rentenansprüchen. Modellierungen der makroökonomischen Effekte mit kurzfristig orientierten Modellen ergeben in der Tat prozyklische Effekte, diese Modellansätze sind dem langfristigen Charakter der privaten Altersvorsorge jedoch völlig unangemessen. Schließlich hat die Finanz- und Wirtschaftskrise 2008/09 an den beiden zentralen Gründen, die für einen Teilumstieg vom Umlage- zum Kapitaldeckungsverfahren sprechen, nichts geändert: erstens der höhere Kapitalstock, der in der langen Frist ein höheres Bruttosozialprodukt ermöglicht, weil die steuerähnliche Belastung der Arbeitnehmer sinkt und die Gesamtfaktorproduktivität steigt; zweitens die gleichmäßigere Belastung der Generationen angesichts des dramatischen demographischen Wandels.

Dieser Beitrag ruft zunächst die wirtschaftstheoretische Diskussion ins Gedächtnis zurück (Abschnitt 2), einschließlich der so genannten Mackenroth-These, die immer wieder fälschlicherweise als Indiz für eine Art Äquivalenz von Umlagesystem und

\footnotetext{
${ }^{1}$ Coppola und Reil-Held (2009).
} 
Kapitaldeckungssystem interpretiert wird (Abschnitt 3). Im empirischen Teil dieses Beitrags gehen wir auf die Renditediskussion (Abschnitt 4) und die makroökonomischen Auswirkungen der Riester-Rente ein (Abschnitt 5). Anzumerken ist natürlich, dass die Riester-Rente als prominentestes individuelles Altersvorsorgeinstrument neben der sonstigen ungeförderten privaten Altersvorsorge und den Betriebsrenten pars pro toto für die gesamte kapitalgedeckte Altersvorsorge steht.

\section{Der theoretische Rahmen}

Das makroökonomische Argument für die Effizienz des Kapitaldeckungsverfahrens (KDV) ist einfach zu verstehen und unter Ökonomen unumstritten: ${ }^{2}$ Das Umlageverfahren (UV) verdrängt Ersparnis durch die Sparillusion der Beitragszahlungen, welche nicht in Investitionen, sondern direkt in den Konsum der älteren Generation fließen. Wird stattdessen im KDV gespart und investiert, entsteht ein höherer Kapitalstock, der die Arbeitsproduktivität und damit das Bruttoinlandsprodukt erhöht. Aus der mikroökonomischen Perspektive spiegelt sich diese höhere Effizienz in den unterschiedlichen Renditen des UV und des KDV wider. In einer stationären Ökonomie identischer Individuen sind die makro- und mikroökonomischen Renditen der beiden Systeme jeweils gleich. Die makro- wie mikroökonomische Rendite ist im KDV die Kapitalrendite und im UV die Wachstumsrate der Lohnsumme, d.h. der Summe aus der Wachstumsrate der Erwerbstätigen und der Rate des arbeitsvermehrenden technischen Fortschritts. Solange kein Schneeballsystem mit explodierender Staatsverschuldung zugelassen wird, ist die Kapitalrendite stets mindestens so hoch wie das Lohnsummenwachstum, und das Umlageverfahren kann nicht rentierlicher sein als das Kapitaldeckungsverfahren.

Dieses klare theoretische Ergebnis ist aus zwei Gründen allerdings graue Theorie. Erstens sind Ökonomien weder stationär noch bestehen sie aus identischen Individuen. Die Nichtstationarität beschert uns immer wieder Phasen, in denen die Rendite des UV höher als die des KDV ist. Dies war zum Beispiel in der Aufbauphase der Bundesrepublik nach dem zweiten Weltkrieg der Fall, als die Kapitalrenditen niedrig und die Arbeitsproduktivitätssteigerungen sehr hoch waren. Zudem ist die Kapitalrendite großen kurzfristigen Schwankungen um einen langfristigen Trend unterworfen. Nicht zuletzt die Finanzkrise 2008 hat uns dies wieder bewusst gemacht. Schließlich gibt es aus

\footnotetext{
${ }^{2}$ Das war nicht immer so. Vgl. Abschnitt 3 und Homburg (1988) für eine klar formulierte Widerlegung der sog. Mackenroth-These.
} 
mikroökonomischer Sicht erhebliche Renditeunterschiede im UV z.B. zwischen Männern und Frauen sowie zwischen Früh- und Spätrentnern. Die volkswirtschaftliche Realität erfordert daher eine subtilere Argumentation, macht den obigen Gedankengang aber nicht falsch.

Wichtiger ist der zweite Grund. Er liegt im Umstand, dass die heutigen Erwerbstätigen nicht die Wahlfreiheit haben, aus dem derzeitigen Umlageverfahren auszutreten und in ein kapitalgedecktes System zu wechseln, weil sie die Renten der gegenwärtigen Rentnergeneration finanzieren müssen. Dieses Übergangsproblem erfordert es, dass eine Generation zweimal einzahlt - für ihre Eltern und für sich selbst. Die Übergangsgeneration muss die impliziten Staatsschulden der ersten Generation zurückzahlen, die nach der Einführung des UV Renten erhielten, ohne volle Beiträge gezahlt zu haben.

Die Literatur hat sich ausgiebig mit diesem Umstellungsproblem beschäftigt. Fenge (1997) bietet eine umfangreiche Übersicht verschiedener Übergangsszenarien im Rahmen neoklassischer Wachstumsmodelle. Diese Literatur konzentriert sich auf die Frage, ob ein intergeneratives Umverteilungsschema existiert, das eine Kompensation der Übergangsgeneration durch die zukünftigen Generationen, die vom Kapitaldeckungsverfahren profitieren werden, ermöglicht. ${ }^{3}$ Die vorgeschlagenen Schemata erfordern eine Kreditaufnahme während der Übergangsperiode. Diese nimmt jedoch Ressourcen in Anspruch und verursacht bei endogenem Arbeitsangebot Wohlfahrstverluste, weil die entstehenden Schulden durch verzerrende Steuern bedient werden müssen. Daher sind die Arbeitsangebotselastizität und das Ausmaß, in dem die Rentenbeiträge als Steuern aufgefasst werden, entscheidende Parameter zur Beurteilung der Wohlfahrtseffekte eines Übergangs.

Werden die Beiträge ganz oder zu einem großen Teil als Steuern aufgefasst, ist ein Übergang zum Kapitaldeckungsverfahren vorteilhaft: alle Generationen können besser gestellt werden („pareto-effizient“). Diese Ansicht dominiert die U.S.-amerikanische Literatur. So haben Feldstein und Samwick (1996) und Kotlikoff (1996) Kalkulationen für die Vereinigten Staaten durchgeführt, die zeigen, dass es bei realistischer Wahl der Parameter möglich ist, Umstellungsstrategien zu entwickeln, die vorteilhaft für alle Generationen sind. Raffelhüschen (1993) sowie Buslei und Kraus (1996) stellen ähnliche Berechnungen für Deutschland vor.

Anders sieht es aus, wenn die Beiträge zum UV nicht als verzerrende Abzüge vom Bruttolohn empfunden werden, sondern als Beiträge, denen 1:1 auch Leistungen gegenüberstehen.

\footnotetext{
${ }^{3}$ Die wichtigsten Fenge (1997) zugrunde liegenden Originalarbeiten sind Breyer (1989), Breyer und Straub (1993), sowie Homburg (1990).
} 
Nimmt man wie Fenge (1995) diese sogenannte Teilhabeäquivalenz an, also das Fehlen jeglicher intragenerativer Umverteilung, und hat die Ausgestaltung der Rentenversicherung keinerlei Effekte auf die Produktionsstruktur, kann man zeigen, dass eine Umstellung vom UV zum KDV nicht pareto-verbessernd sein kann, da eine Kompensation der Übergangsgeneration in diesem Fall den Vorteil des KDV exakt wieder zunichte macht. Ähnliche Argumente finden sich bereits bei Diamond (1965) und Aaron (1966), und werden von Brunner (1994, 1996) und Pestieau (1994) wieder aufgegriffen.

Die Vorteilhaftigkeit einer (Teil-)umstellung von UV auf KDV hängt daher von zwei empirischen Fragen ab. Erstens, inwieweit blicken die Arbeitnehmer durch den „Schleier“ der Sozialbeiträge durch und fassen sie als Versicherungsprämien auf und nicht als steuerähnliche Abzüge vom Bruttolohn? Zweitens, inwieweit gibt es Rückkopplungen des Rentensystems auf den Produktionssektor?

Umfrageergebnisse unter jungen Arbeitnehmern („Erwarten Sie, dass Sie Ihren Sozialversicherungsbeiträgen entsprechende Leistungen erhalten werden?“) zeigen, dass nur wenige an eine Äquivalenz von Beiträgen und Leistungen glauben und eine Mehrheit die Sozialversicherungsbeiträge als Steuern auffasst. ${ }^{4}$ Diese Umfrageergebnisse finden in der Flucht in den nicht sozialversicherungspflichtigen Bereich des Arbeitsmarktes eine deutliche Bestätigung. ${ }^{5}$ Auch institutionell ist die Annahme der Teilhabeäquivalenz fragwürdig, da es in Deutschland zwar keine so starke intragenerative Umverteilung zwischen Reich und Arm wie in den USA gibt, dafür aber eine umso stärkere intragenerative Umverteilung zwischen Frührentnern und Spätrentnern, zwischen Männern und Frauen und dementsprechend zwischen verheirateten und alleinstehenden Arbeitnehmern. ${ }^{6}$ Schließlich ist aus mikroökonomischer Perspektive der Renditeunterschied für das Arbeitsangebotsverhalten maßgeblich: Unterscheidet sich die Rendite des KDV deutlich von der impliziten Rendite des $\mathrm{UV}^{7}$ wird der Arbeitnehmer versuchen, aus dem für ihn weniger rentierlichen System auszuweichen, weil er dem Übergangsproblem aus seiner mikroökonomischen Perspektive in einer Art Trittbrettfahrereffekt entweichen kann, in dem er die Finanzierung der Rente der laufenden älteren Generation den Anderen überlässt. Börsch-Supan und Reil-Held (2001) argumentieren, dass aus der Sicht eines jungen Arbeitnehmers etwa drei Viertel des Rentenversicherungsbeitrags Steuern sind. Gasche (2008) berechnet einen impliziten

\footnotetext{
${ }^{4}$ Vgl. die Zusammenstellung in DIA (1999).

${ }^{5}$ Der Anstieg der geringfügig Beschäftigten und der (Schein-)Selbständigen ist in Schnabel (1998a) dokumentiert. Über Schwarzmarkttransaktionen ist naturgemäß wenig bekannt.

${ }^{6}$ Vgl. Schnabel (1998b).

${ }^{7}$ Vgl. auch Abschnitt 4.
} 
Steueranteil an den Beitragszahlungen des gesamten Erwerbslebens zwischen ca. 40\% und 60\%, je nachdem ob man berücksichtigt, dass die Rentenversicherung auch Erwerbsunfähigkeits- und Hinterbliebenenrenten zahlt und je nachdem ob man Männer oder Frauen betrachtet. Gasche (2009) zeigt, dass in der Beitragszahlung eines Jahres z.B. für 25bis 29-Jährige ein impliziter Steueranteil in der Spannbreite zwischen ca. 45\% (Frauen Ost) und 55\% (Männer West) steckt.

Die Rückkopplungseffekte des Rentensystems auf den Produktionssektor und Abweichungen von der fixen neoklassischen Produktionsstruktur, die den in Fenge (1997) zusammengefassten Arbeiten unterliegt, sind groß. Fenge nimmt an, dass der Übergang vom UV zum KDV die Arbeitsproduktivität erhöht, aber im gleichen Maß die Kapitalproduktivität senkt, so dass die totale Faktorproduktivität unberührt bleibt. Dagegen betont eine Reihe empirischer Untersuchungen der Weltbank eine durch die Einführung des KDV erhöhte totale Faktorproduktivität. $^{8}$ Diese entsteht durch die Reduktion des informellen Sektors (Schwarzarbeit bzw. geringfügige Beschäftigungsverhältnisse) zugunsten des produktiveren formellen Sektors im Arbeitsmarkt, durch eine höhere Effizienz der Kapitalmarktinstitutionen, die ihrerseits aus der Stärkung institutioneller Anleger (z.B. professionelle Pensionsfonds) folgt, und durch eine Erhöhung der Kapitalproduktivität mittels verbesserter Eigentümerkontrolle („,corporate governance“), die wiederum durch den Marktdruck der Pensionsfonds zustande kommt. ${ }^{9}$ Studien, die sich auf hinreichend lange Zeitreihen stützen können, sind selten; viele beruhen auf der chilenischen Erfahrung und zeigen stark positive Rückkopplungen auf die totale Faktorproduktivität, das Sozialproduktswachstum und die Ersparnis. ${ }^{10}$ Börsch-Supan, Köke und Winter (2005) können die positiven Rückkopplungen institutioneller Investoren auf die Kapitalproduktivität auch für deutsche und britische Unternehmen nachweisen.

Pestieau und Possen (2000) betonen Verzerrungen, die aus dem Wechselspiel von Liquiditätsbeschränkungen und Heterogenität im Risikoverhalten entstehen. Das Umlageverfahren zwingt de facto die Haushalte, einen großen Teil ihres Vermögens in Staatsschuld anzulegen. Falls die Haushalte aber risikofreudiger sind, jedoch ihr Gesamtvermögen nicht weiter ausdehnen wollen, kommt es zu Verzerrungen der Portefeuillestruktur in der Ökonomie, die die Wohlfahrt und das Sozialprodukt verringern.

\footnotetext{
${ }^{8}$ Vgl. etwa World Bank (1994), Corsetti (1994), Cosetti und Schmidt-Hebbel (1995), Holzmann (1997).

${ }^{9}$ Konkrete Beispiele finden sich in Börsch-Supan (1998), Regressionsanalysen zum Zusammenhang zwischen Kapitalmarktinstitutionen und Produktivität in Levine und Zervos (1998) sowie Rajan und Zingales (1998).

${ }^{10}$ Vgl. Diamond (1994), Holzmann (1997), Schmidt-Hebbel (1998).
} 
In gewisser Weise ist dieses Argument mit dem Verteilungsargument von Sinn (2000) verwandt, der betont, dass der Hauptgrund für einen (Teil-)umstieg vom UV auf das KDV nicht in der höheren Rendite des KDV liegt, sondern in der Tatsache, dass nur eine KDV die Lasten der demographischen Entwicklung über die Generationen verteilen kann. Denn durch die Alterung entstehen enorme Lasten für zukünftige Generationen, sei es im Steuersystem oder in den umlagefinanzierten Sozialsystemen. Eine in Relation immer kleiner werdende Schar jüngerer Nettozahler muss für die relativ wachsende Zahl der älteren Nettoempfänger (z.B. Rentner) aufkommen. Die Belastung der Jungen und zukünftigen Generationen steigt mithin an. Die Kapitaldeckung ist nun dazu geeignet, diese Lasten anders zu verteilen - über die Zeit und über die Generationen. Bildet beispielsweise die derzeit noch im Erwerbsleben stehende Babyboomer-Generation für ihr eigenes Alter Kapital, sorgt sie für sich selbst vor. In dem Maße wie diese Gruppe für sich selbst vorgesorgt hat, ist sie im Alter nicht auf die Versorgung durch die dann Jungen angewiesen. Die Jungen werden von dieser Last befreit. Sie wird interpersonell, intertemporal und intergenerativ hin zur Babyboomer-Generation verschoben, damit gleichmäßiger. Eine gleichmäßige Belastung der Generationen ist aber sowohl aus Nutzen- als auch Liquiditätsaspekten paretoverbessernd im Vergleich zu einer ungleichmäßigen Belastung. Diese Eigenschaft der intergenerativen und intertemporalen Lastumverteilung und damit der Beitrag der privaten Altersvorsorge zur Bewältigung der demographischen Herausforderungen ist ein weiterer wichtiger Grund, warum die private kapitalgedeckte Altersvorsorge von großer Bedeutung ist.

Zusammengefasst gibt es also zwei gewichtige Gründe, die für einen (Teil-)umstieg vom UV zum KDV sprechen: erstens, der höhere Kapitalstock, der trotz des Übergangsproblems entsteht und in der langen Frist ein höheres Bruttosozialprodukt ermöglicht, weil die steuerähnliche Belastung der Arbeitnehmer sinkt und die Gesamtfaktorproduktivität steigt; zweitens, die gleichmäßigere Belastung der Generationen angesichts des dramatischen demographischen Wandels. An beiden Gründen hat die Finanz- und Wirtschaftskrise 2008/09 nichts geändert.

\section{Die Mackenroth-These}

Oft wird die Vorstellung propagiert, dass sich Umlagesystem und Kapitaldeckungssystem gar nicht so sehr unterscheiden, weil letztlich alle Rentenzahlungen aus dem Volkseinkommen eines Jahres aufgebracht werden müssen, egal ob es sich um kapitalgedeckte Renten oder um umlagefinanzierte Renten handelt (Mackenroth-These). 
Diese These wird zumindest falsch interpretiert. Denn es ist zwar richtig, dass alle Rentenzahlungen einer Periode letztlich aus dem Volkseinkommen finanziert werden müssen. Aber eine häufige Fehlinterpretation übersieht, dass bei einem Umlagesystem dieses Volkseinkommen nicht gleich dem Volkseinkommen im Kapitaldeckungssystem sein muss. ${ }^{11}$ Bildlich gesprochen: Die Größe des „Kuchens“, der verteilt werden kann, unterscheidet sich. Er ist bei einem Kapitaldeckungsverfahren, wie in Abschnitt 2 diskutiert wurde, tendenziell größer.

Eine ausführliche mathematische Ableitung findet sich z.B. in Homburg (1988). Man kann dies aber auch intuitiv anhand der einfachen Ex-Post-Identität zeigen, dass die volkswirtschaftliche Ersparnis den Investitionen plus Außenbeitrag entsprechen muss: $S_{P R}+S_{S T}+S_{U}=I+X-M$

Eine höhere private Ersparnis $S_{P R}$ (bzw. eine höhere Ersparnis des Staates $S_{S T}$ oder der Unternehmen $S_{U}$ ) geht mit höheren Investitionen $I$ und oder einem höheren Außenbeitrag $X$-M (=Exporte minus Importe) einher. Eine Erhöhung der Investitionen $I$ erweitert den volkswirtschaftlichen Kapitalstock und führt damit zu einer höheren Produktivität und damit zu einem höheren Sozialprodukt und einem höheren Volkseinkommen.

Gegen diesen Wirkungszusammenhang wird eingewandt, dass es ja auch unproduktive Investitionen gebe, was man am Beispiel der Sowjetunion gesehen habe. ${ }^{12}$ Dieser Vergleich ist falsch, da das Planwirtschaftssystem der Sowjetunion nur sehr wenig mit einem marktwirtschaftlichen System gemein hatte. In der Sowjetunion gab es mangels Wettbewerb nur einen beschränkten Anreiz, produktive Investitionen durchzuführen; Fehlinvestitionen wurden nicht vom Markt bestraft. In einem marktwirtschaftlichen System dagegen werden ex ante unrentable Investitionsprojekte nicht durchgeführt, weil man damit am Markt nicht bestehen könnte. $^{13}$

Als zweite Kritik an dem oben beschrieben Zusammenhang wird vorgebracht, dass es gar nicht zu höheren Investitionen im Inland kommt, sondern dass Kapital ins Ausland abfließt bzw. die Erlöse aus den Exportüberschüssen des Exportweltmeisters Deutschland im Ausland verbleiben. Dagegen kann man zunächst vorbringen, dass weder ausschließlich $I$ steigen wird noch ausschließlich $X-M$ steigen wird. Es wird vielmehr eine Mischung aus beiden geben. Zudem bedeutet die Tatsache, dass Mittel ins Ausland fließen, nicht, dass damit das Geld

\footnotetext{
${ }^{11}$ Z.B. Logeay et al. (2009).

${ }^{12}$ Vgl. Meinhardt et al. (2009), S. 54.

${ }^{13}$ Dies schließt natürlich nicht aus, dass in beiden Systemen ex ante als rentabel angesehene Investitionen sich ex post als unrentabel erweisen.
} 
verloren ist. Vielmehr kann im Ausland Kapital gebildet werden, z.B. Produktionsstätten gekauft oder errichtet werden. Das dort geschaffene Einkommen kann dann wieder zurück ins Inland fließen und hier das Volkseinkommen erhöhen, also den Kuchen vergrößern. ${ }^{14}$

Dagegen wird wiederum argumentiert, dass gerade die Finanzkrise gezeigt habe, wie im Ausland angelegtes Kapital verpuffen kann, z.B. wenn man in amerikanische Schrottimmobilen („Subprime Mortgages“) investiert habe. Zudem bestehe bei Auslandsanlagen ein Wechselkursrisiko, das ebenfalls zum Verlust führen könne. ${ }^{15}$

Natürlich sind die Vermögensverluste durch die Finanzkrise nicht zu leugnen. Allerdings darf man bei der Beurteilung der kapitalgedeckten Altersvorsorge keine kurzfristige Perspektive einnehmen, sondern muss angesichts der langen Laufzeit eine mittel- und langfristige Betrachtung vornehmen, vgl. den folgenden Abschnitt. Langfristig hat Deutschland sehr stark von der Kapitalbildung im Ausland profitiert, und wird dies angesichts des demographischen Wandels in Zukunft noch mehr tun. ${ }^{16}$

Ein Wechselkursrisiko ist bei Auslandsanlagen außerhalb der Europäischen Währungsunion (EWU) immer vorhanden. Wenn es allerdings eine solche große Bedeutung hätte, wie dies suggeriert wird, dürfte es gar keinen Außenhandel geben und keinen Kapitalexport, weil sich ein Auslandsengagement nie lohnen würde. Vielmehr ist es so, das innerhalb der EWU das Wechselkursrisiko überhaupt nicht besteht und bei den meisten anderen Ländern beherrschbar ist. Dafür gibt es zahlreiche Kurssicherungsinstrumente am Kapitalmarkt.

Freilich kann man auch argumentieren, dass eine höhere Ersparnis $S_{p r}$ letztlich für die Finanzierung einer größeren negativen Ersparnis des Staates, also einer höheren Staatsverschuldung verwendet werden kann. Beruht ein kapitalgedecktes System nur auf Staatschulden des eigenen Landes, hätte man ein (Pseudo-)Kapitaldeckungssystem, das dem Umlagesystem äquivalent ist. Umso wichtiger ist es, dass die Kapitaldeckung nicht nur auf inländischen Staatsschulden beruht, sondern zumindest auch auf der Beteiligung am Fremdund Eigenkapital von in- und ausländischen Unternehmen.

Die Mackenroth-These ist - jedenfalls in der immer wieder vertretenen Form, die eine Art Äquivalenz von Umlagesystem und Kapitaldeckungssystem propagiert - falsch, weil sie

\footnotetext{
${ }^{14}$ Dass Deutschland netto betrachtet derzeit ein Kapitalexporteur ist, heißt ja gerade, dass aus dem Ausland Einkommen ins Inland fließt.

${ }^{15}$ Vgl. Logeay et al. (2009), S. 3.

16 Börsch-Supan, A., Ludwig, A. und J. Winter (2006).
} 
ignoriert, dass eine Volkswirtschaft dynamisch ist, also Wachstum erzeugt, das je nach Umlage- oder Kapitaldeckungsverfahren unterschiedlich ausfällt, und weil sie ignoriert, dass in einer offenen Volkswirtschaft Kapitalexporte zu einem Zuwachs an Volkseinkommen führen.

\section{Renditevergleiche}

Die Diskussion pro und contra (Teil-)umstieg zum Kapitaldeckungsverfahren wird häufig von Renditevergleichen dominiert. Dies ist irreführend, da die zentralen Vor- und Nachteile der beiden Finanzierungsverfahren auf einer ganz anderen Ebene liegen, wie in Abschnitt 2 betont wurde, nämlich auf den unterschiedlichen Implikationen für in- und ausländische Investitionen und damit das Wachstum des Volkseinkommens (Homburg, 1988; BörschSupan et al. 2002, 2003 und 2006), sowie den unterschiedlichen Möglichkeiten, Ressourcen zwischen Generationen zu verteilen (Sinn, 2000).

Auf zwei Punkte soll dennoch hier eingegangen werden, weil sich in ihnen immer wieder geäußerte Fehlvorstellungen konzentrieren: zum einen, welche Arten von Anlagen die beiden Verfahren kennzeichnen und wie sie abgegrenzt werden, zum zweiten der Einfluss der Finanz- und Wirtschaftskrise auf die Renditen der beiden Finanzierungsverfahren.

Häufig wird angemerkt, dass man für den Renditevergleich als Kapitalmarktrendite nur die Rendite sicherer Staatsanleihen verwenden dürfe. ${ }^{17}$ Dies ist falsch, denn es unterstellt, dass das Umlagesystem selbst völlig sicher ist und keine Risiken in sich birgt, vor allem nicht insolvent werden kann. Das stimmt so nicht; das Blüm'sche Diktum hat nur sehr grundsätzlichen Charakter und seinem Verkünder viel Spott eingebracht. Zunächst gibt es das demographische Risiko. Rentenansprüche sind wiederholt gekürzt worden, nicht nur durch die großen Rentenreformen 1992, 2001 und 2004 in der Notwendigkeit, frühere Versprechen der demographischen Realität anzupassen, sondern auch in kleinen, vielen Menschen bislang verborgenen Schritten, wie der heute weit geringeren Anrechnung von Ausbildungszeiten als es früher der Fall war. Zudem ist die Sicherheit von Rentensystemen immer auch eine politische. So können z.B. aus der demographischen Entwicklung resultierende Finanzierungsprobleme der Rentenversicherung, die zu massiven Beitragssatzsteigerungen führen, die Akzeptanz und damit letztlich den Bestand eines Rentensystems gefährden. Ein durch diese Akzeptanzprobleme drohender Systemwechsel, z.B. hin zu einem Grundrentensystem, würde die Rendite für viele auf Null senken oder sogar negativ werden

\footnotetext{
${ }^{17}$ Z.B. Logeay et al. (2009).
} 
lassen. Das demographische Risiko und das politische Risiko gehen beim Umlagesystem also Hand in Hand. ${ }^{18}$

Nicht korrekt ist auch das Argument, Renditevergleiche würden dem Versicherungscharakter der Rentenversicherung nicht gerecht. Die biometrischen Risiken Langlebigkeit, Invalidität, Verwitwung und Verwaisung können auch im Kapitaldeckungsverfahren abgedeckt werden, ähnlich wie dementsprechende private Invaliditäts- und Lebensversicherungen, die auf dem Versicherungsmarkt erhältlich sind. Entweder müssen die entsprechenden Kosten in die Renditeberechnungen der privaten Altersvorsorge einbezogen oder diese Leistungen bei der Berechnung der Rendite der Gesetzlichen Rentenversicherung als renditesteigernd berücksichtigt werden (vgl. z.B. Gasche, 2008).

Auch über den Einfluss der Finanz- und Wirtschaftskrise 2008/09 gibt es große Fehleinschätzungen. Zum einen sind die Renditeverluste beim Altersvorsorgevermögen geringer als es in der breiten Öffentlichkeit dargestellt wird. Oft beherrscht der massive Einbruch des DAX um etwa 40\% im Jahr 2008 zumindest die Presse. Aktien machen jedoch nur einen geringen Anteil der Anlagen für die private Altersvorsorge aus, und zudem werden selbst massive Schocks über die lange Zeit, in der die Altersvorsorgeprodukte akkumuliert werden, stark geglättet. Börsch-Supan, Gasche und Ziegelmeyer (2009) berechnen auf Basis der SAVE-Daten, dass der durchschnittliche relative Verlust des Altersvorsorgevermögens über alle Haushalte gerechnet bei etwa $2 \%$ liegt. Dies ist deutlich niedriger als der durchschnittliche relative Verlust im Finanzvermögen (ca. 4,3\%), der seinerseits deutlich unter den DAX-Verlusten liegt. Selbst bei einer groben auf makroökonomischen Aggregaten beruhenden Schätzung liegen die Verluste des Altersvorsorgevermögens unter 3\% und die des gesamten Finanzvermögens unter 8,5\%. Schreibt man diese Vermögensverluste differenziert für die Geburtsjahrgänge 1940 bis 1990 über das gesamte Erwerbsleben bis zum Rentenalter fort, ergeben sich daraus je nach Geburtsjahrgang Renditeeinbußen von bis zu 0,1 Prozentpunkten für das Altersvorsorgevermögen und bis zu 0,2 Prozentpunkten für das Finanzvermögen. Im Übrigen werden diese Verluste, in seltsamen Kontrast zu der in der Presse geschilderten Meinung, auch von den Haushalten selbst als so gering eingeschätzt, wie die Auswertungen von Börsch-Supan, Bucher-Koenen, Gasche und Ziegelmeyer (2009) zeigen. ${ }^{19}$

\footnotetext{
${ }^{18}$ Auch im Kapitaldeckungssystem gibt es politische Risiken wie das der Enteignung und einer enteignenden Besteuerung. Beides findet in Deutschland seine Grenzen im Eigentumsschutz.

${ }^{19}$ Ähnlich Benz et al. (2009). Die OECD (2009) zeigt, dass gerade in Deutschland die Verluste besonders niedrig waren.
} 
Diesen Verlusten im kapitalgedeckten Teil der Altersvorsorge steht nicht etwa ein von der Finanz- und Wirtschaftskrise unberührtes Umlageverfahren gegenüber. Auch hier sind Verluste zu verzeichnen. Insgesamt zeigen sich weit reichende Parallelen zu den Auswirkungen der Finanzkrise auf die Gesetzliche Rentenversicherung, denn die dort stattfindende Abwertung der Entgeltpunkte durch Arbeitslosigkeit, Kurzarbeit und Lohnstagnation oder -senkung kann ebenfalls als Vermögensverlust interpretiert werden, der alle Jahrgänge permanent trifft, die zum Zeitpunkt der Krise im Erwerbsleben standen.

So berechnen Börsch-Supan, Gasche und Wilke (2009), wie stark sich die Arbeitslosigkeit, Kurzarbeit und Lohnstagnation oder -senkung im Verlaufe der Krise auf die zukünftigen Rentenansprüche ausgewirkt haben. Die Abwertung der Entgeltpunkte liegt in der Höhe von 5\% bis 8\%, je nach Verlauf der Krise im zweiten Halbjahr 2009 und 2010. Dies ist also größenordnungsmäßig mit den Verlusten von 2\% (Altersvorsorgevermögen) und 4\% (Finanzvermögen) vergleichbar. Der Verlust der impliziten Rendite der Gesetzlichen Rentenversicherung durch die Krise wurde je nach Szenario zwischen 0,1 und 0,3 Prozentpunkten quantifiziert, was dementsprechend ähnlich $\mathrm{zu}$ den Verlusten von 0,1 Prozentpunkten (Altersvorsorgevermögen) und 0,2 Prozentpunkten (Finanzvermögen) ist. Beide Analysen zeigen die stärkste Betroffenheit bei den Rentnerjahrgängen und den rentennahen Jahrgängen, weil diese Jahrgänge zum Zeitpunkt der Krise schon viel Vermögen angesammelt hatten, entweder in Form von Entgeltpunkten oder in Form von Finanzkapital.

Somit sind die Renditen keines der beiden Altersvorsorgesysteme von der Krise verschont geblieben. Sowohl Kapitaldeckungs- wie Umlageverfahren sind betroffen. Für beide Vorsorgearten hält sich die Betroffenheit jedoch in Grenzen.

\section{Makroökonomische Auswirkungen der Riester-Reform}

Die für Finanz- und Wirtschaftskrisen typische Erhöhung der Sparquote wirkt prozyklisch und ist daher höchst problematisch, weil sie die Krise vertieft. Die Lehrbuchstrategie volkswirtschaftlichen Krisenmanagements besteht daher aus einem (über-)kompensierenden Anstieg der konsumtiven Staatsausgaben, der mittels Staatsverschuldung finanziert wird.

In diesem Zusammenhang ist auch die Riester-Rente erst als konjunktur-, dann als wachstumsschädlich bewertet worden, vgl. z.B. die Studie des Instituts für Makroökonomie und Konjunkturforschung (IMK). ${ }^{20}$ Diese Argumentation, die darauf beruht, Sparen

\footnotetext{
${ }^{20}$ Meinhardt et al. (2009). Zusammengefasst bei Logeay et al. (2009) (IMK Report Nr. 43).
} 
grundsätzlich als konjunkturschädlich anzusehen, ist sowohl logisch als auch methodisch problematisch.

Zum einen besteht der Zweck der Riester-Rente in erster Linie darin, die durch die Rentenreformen 2001 und 2004 geschaffenen Rentenlücken bei der gesetzlichen Rente durch die zusätzliche private Altersvorsorge langfristig zu schließen. Damit diese Lücke geschlossen wird, muss zusätzlich zur bisherigen Ersparnis langfristig Kapital gebildet werden. Diese langfristige Absicht hat also nichts mit prozyklischem Verhalten zu tun. Der Effekt einer höheren Ersparnis durch die Riester-Rente ist explizit erwünscht, er muss stetig und konjunkturunabhängig sein, denn sonst würde die durch die graduelle Senkung des Rentenniveaus bestehende Rentenlücke nicht geschlossen. Zusätzliche Ersparnis aufgrund der Riester-Reform ist daher nichts Schlechtes, sondern im Gegenteil ein Erfolg. Das MEA konnte anhand der Auswertung der SAVE-Umfrage Indizien für diesen Erfolg finden. ${ }^{21}$

Daher sind auf kurzfristigen Konjunkturmodellen basierende Untersuchungen wie die oben zitierte ungeeignet, wirtschafts- und sozialpolitische Wertungen zur Riester-Rente zu liefern. Hinzu kommen methodische Bedenken:

Erstens ist bei den Berechnungen des IMK das Riester-Sparen nicht explizit abgebildet, sondern nur als Effekt eines allgemeinen Anstiegs der Sparquote. Davon ist jedoch nur ein Teil auf das Riester-Sparen selbst zurückzuführen.

Zweitens wird der Anstieg der Sparquote zwischen 2001 und 2008 hauptsächlich durch die Einkommensverteilung und durch das zusätzliche Vorsorgesparen durch die Riester-Reform erklärt. Die Änderung der Einkommensverteilung wird mit der Änderung der Gewinnquote abgebildet, was nicht korrekt ist, weil es die Veränderung der Verteilung abhängig Beschäftigter ausblendet - gerade die Divergenz zwischen Niedriglohngruppen und Managergehältern wurde ja jüngst immer wieder thematisiert. ${ }^{22}$ Das erhöhte Vorsorgesparen wird mit einem exogen angenommenen trendmäßigen Anstieg der Sparquote abgebildet. Letztlich erklärt also ein trendmäßiger Anstieg der Sparquote den Anstieg der Sparquote. Dass diese Trendvariable für die Entwicklung der Sparquote signifikant ist, vermag also nicht verwundern. Es kommt das heraus, was vorher hineingesteckt wurde.

Drittens, und wohl am wichtigsten, ist der fehlspezifizierte Zusammenhang zwischen kurzfristigen Konjunktur- und langfristigen Wachstumseffekten. Ganz generell zeigen alle langfristigen Wachstumsmodelle einen Anstieg des BIP bei einem stärkeren Anteil

\footnotetext{
${ }^{21}$ Coppola und Reil-Held (2009).

${ }^{22}$ Wissenschaftlicher Beirat beim Bundesministerium für Wirtschaft und Technologie (2009).
} 
kapitalgedeckter Renten. ${ }^{23}$ Auch in dem vom MEA verwendeten Wachstumsmodell, das die Zusammenhänge zwischen Produktionsfunktion und Konsum- bzw. Sparfunktion wechselseitig berücksichtigt, führt die höhere Ersparnis langfristig zu einem höheren Kapitalstock und damit auf einen höheren Wachstumspfad, also zu einer positiven BIPEntwicklung. ${ }^{24}$

Empirische Belege dazu finden sich z.B. bei Diamond (1994), Holzmann (1997) und Schmidt-Hebbel (1998). Nach Berechnungen des MEA wirkt sich auch die Riester-Reform positiv auf das BIP aus, obwohl sie ja nur einen kleinen Anteil der Gesamtrentenfinanzierung vom Umlage- zum Kapitaldeckungsverfahren umwandelt. ${ }^{25}$

Ein gegensätzliches Ergebnis, also das eines negativen Effekts der Riester-Reform auf das Wirtschaftswachstum, ist weniger den konkreten Wirkungen der Riester-Reform geschuldet, sondern beruht vielmehr darauf, dass in dem zur Abschätzung der Wirkungen verwendeten Konjunkturmodell ganz generell eine Erhöhung der Sparquote die Wirtschaftsentwicklung negativ beeinflusst. Wird in einem Modell die Produktion und damit der Zusammenhang zwischen Ersparnis, Kapitalbildung und höherer Produktivität nicht explizit modelliert, ist Sparen immer nachteilig für die BIP-Entwicklung, weil es den Konsum und damit die gesamtwirtschaftliche Nachfrage beeinträchtigt.

Dies widerspricht jedoch der Wachstumstheorie, bei der Investitionen in Human- und Realkapital die wesentlichen Wachstumsfaktoren sind. Keynesiansche Konjunkturmodelle sind auf das langfristige Wachstum nicht anwendbar; sie beschreiben lediglich den Einfluss erhöhter (Staats-)Ausgaben bei brachliegenden Ressourcen, vor allem Massenarbeitslosigkeit, und die Übertragung von kurzfristigen Ergebnissen auf das langfristige Wachstum ist methodisch illegitim.

Bei einer eher langfristigen Sichtweise, also aus der Wachstumsperspektive, ist Sparen nicht per se schlecht, sondern ganz im Gegenteil: Durch die volkswirtschaftliche Ersparnis werden Investoren die Mittel bereitgestellt, um zu investieren und damit Kapital z.B. in Form von Maschinen zu bilden. Dieses zusätzliche Kapital erhöht die volkswirtschaftliche Produktion und damit das BIP. Sparen hat also einen positiven Wachstumseffekt. Ohne Produktion kann man kein Wachstum analysieren (Solow, 1956).

Viertens werden in dem verwendeten Konjunkturmodell Anreizeffekte, die von den Beitragszahlungen ausgehen, nicht berücksichtigt. So haben die Beiträge zur Gesetzlichen

\footnotetext{
${ }^{23}$ U.a. Kotlikoff (1996), Altig et al. (2001), Rios-Rull (2001), Brooks (2003).

${ }^{24}$ Z.B. Börsch-Supan et al. (2003 und 2006), am klarsten in Börsch-Supan and Ludwig (2009).

${ }^{25}$ Vgl. Börsch-Supan, Ludwig und Reil-Held (2004).
} 
Rentenversicherung zumindest teilweise einen Lohnsteuercharakter, der einen negativen Effekt auf das sozialversicherungspflichtige Arbeitsangebot (Ausweichen in die Schwarzarbeit, in die Selbständigkeit oder in die Freizeit) ausübt. Will man die Auswirkungen der Riester-Reform abgreifen, muss man die Wirkungen der höheren Beiträge in der Rentenversicherung den Wirkungen der Beitragszahlungen in einen Riester-Vertrag gegenüberstellen. Es ist zu vermuten, dass die Menschen, ihre Beitragszahlung in einen Riester-Vertrag weniger als Steuer auffassen als die Zahlungen zur GRV. Denn beim RiesterVertrag gibt es einen unmittelbareren Zusammenhang zwischen den gezahlten Beiträgen und der Rentenleistung. Man kann diesen Zusammenhang quasi auf dem Kontoauszug sehen. Bei der GRV ist dieser Zusammenhang zwar auch vorhanden, aber er ist diffuser, da man heute Beiträge leistet im Vertrauen darauf, dass zukünftige Rentnergenerationen die mit diesen Beiträgen erworbenen Rentenansprüche bedienen. Zudem ist die Umverteilungskomponente in der GRV größer, was den Zusammenhang zwischen Beiträgen und Leistungen zusätzlich lockert. Ist also der Steuercharakter der GRV-Beiträge tendenziell höher als der der RiesterBeiträge, dann würde eine Eins-zu-Eins-Substitution zwischen GRV-Beiträgen und RiesterBeiträgen die negativen Effekte auf das Arbeitsangebot vermindern und für sich genommen positive Wachstumseffekte haben.

\section{Fazit}

Die Finanz- und Wirtschaftskrise 2008/09 gibt keinen Anlass zu einer grundsätzlichen Neuordnung des von Walter Riester eingeführten Mehrsäulenmodells der deutschen Altersvorsorge. Der mittlere krisenbedingte Verlust des Altersvorsorgevermögens liegt in der gleichen Größenordnung wie der Verlust an gesetzlichen Rentenansprüchen. Modellierungen der makroökonomischen Effekte mit kurzfristig orientierten Modellen ergeben in der Tat prozyklische Effekte, diese Modellansätze sind dem langfristigen Charakter der privaten Altersvorsorge jedoch unangemessen. Auch die beiden zentralen Gründe, die für einen Teilumstieg vom Umlage- zum Kapitaldeckungsverfahren sprechen, bleiben von der Finanzund Wirtschaftskrise 2008/09 unberührt. Der höhere Kapitalstock im Kapitaldeckungsverfahren ermöglicht in der langen Frist ein höheres Bruttosozialprodukt, weil die steuerähnliche Belastung der Arbeitnehmer sinkt und die Gesamtfaktorproduktivität steigt. Zudem ist das „Parken“ von Ressourcen auf dem Kapitalmarkt die einzige Möglichkeit, eine gleichmäßigere Belastung der Generationen angesichts des dramatischen demographischen Wandels zu erzielen. 


\section{Literatur}

Aaron, H. (1966): The Social Insurance Paradox, Canadian Journal of Economics and Political Science, Vol. 32, No. 2, 371-74.

Altig, D., Auerbach, A.J., Kotlikoff, L.J., Smetters, K.A. und J. Walliser (2001): Simulating fundamental tax reform in the United States, American Economic Review, Vol. 91, No. 2, 574-595.

Benz, T., Raffelhüschen, B. und J. Vatter, (2009): Finanzmarktkrise und Altersvorsorge - Wie groß sind die Verluste wirklich? Deutsches Institut für Altersvorsorge, Köln.

Börsch-Supan, A. (1998): Capital's Contribution to Productivity and the Nature of Competition, Brookings Papers on Economic Activity, Microeconomics, Vol. 1998, 205-248.

Börsch-Supan, A. und A. Reil-Held (2001): How much is Transfer and how much Insurance in a Pay-As-You-Go System? The German Case, Scandinavian Journal of Economics, Vol. 130, No.3, 505-524.

Börsch-Supan, A., Ludwig, A. und J. Winter (2002): Aging and International Capital Flows, in: A. J. Auerbach and H. Herrmann (Hrsg.): Ageing, Financial Markets and Monetary Policy, Springer-Verlag, Berlin [u.a], 55-83.

Börsch-Supan, A., Heiss, F., Ludwig, A. und J. Winter (2003): Pension Reform, Capital Markets, and the Rate of Return, German Economic Review, Vol. 4, Issue 2, May 2003, 151-181.

Börsch-Supan, A., Ludwig, A. und A. Reil-Held (2004): Hochrechnungsmethoden und Szenarien für gesetzliche und private Renteninformationen, MEA Diskussionspapier 049-04, Mannheimer Forschungsinstitut Ökonomie und Demographischer Wandel (MEA), Universität Mannheim.

Börsch-Supan, A., Köke, J. und J. Winter (2005): Pension reform, savings behavior, and capital market performance, Journal of Pension Economics and Finance, Vol. 4, No. 1, 87-107.

Börsch-Supan, A., Ludwig, A. und J. Winter (2006): Ageing, Pension Reform and Capital Flows: A Multi-Country Simulation Model, Economica, Vol. 73, No. 292, 625-658.

Börsch-Supan, A. und A. Ludwig (2009): Old Europe ages: Reforms and Reform Backlashes, forthcoming in J. Shoven (Hrsg.), Demography and the Economy, University of Chicago Press, Chicago.

Börsch-Supan, A., Gasche, M. und C. Wilke (2009): Auswirkungen der Finanzkrise auf die Gesetzliche Rentenversicherung, ihre Beitragszahler und ihre Rentner, MEA-Studie Nr. 9, Mannheim.

Börsch-Supan, A., Bucher-Koenen, T., Gasche, M. und M. Ziegelmeyer (2009): Deutsche Privathaushalte in der Finanz- und Wirtschaftskrise - Betroffenheit und Reaktionen, MEA-Studie Nr. 10., Mannheim.

Börsch-Supan, A., Gasche, M. und M. Ziegelmeyer (2009): Auswirkungen der Finanzkrise auf die private Altersvorsorge, MEA Diskussionspapier 193-09, Mannheimer Forschungsinstitut Ökonomie und Demographischer Wandel (MEA), Universität Mannheim. 
Breyer, F. (1989): On the Intergenerational Pareto-Efficiency of Pay-As-You-Go Financed Pension Systems, Journal of Institutional and Theoretical Economics, Vol. 145, No. 4, 643-658.

Breyer, F. und M. Straub (1993): Welfare Effects of Unfunded Pension Systems When Labor Supply is Endogenous, Journal of Public Economics, Vol. 50, No. 1, 77-91.

Brooks, R. (2003): Population aging and global capital flows in a parallel universe, IMF Staff Papers, Vol. 50, No. 2, 200-221.

Brunner, J. (1994): Redistribution and the Efficiency of the Pay-as-you-go Pension System, Journal of Institutional and Theoretical Economics, Vol. 150, No. 3, 511-523.

Brunner, J. (1996): Transition from a Pay-as-you-go to a Fully Funded Pension System: The Case of Differing Individuals and Intragenerational Fairness, Journal of Public Economics, Vol. 60, No.1, 131-146.

Buslei, H, und F. Kraus (1996): Wohlfahrtseffekte eines graduellen Übergangs auf ein niedrigeres Rentenniveau, in: Steiner V. und K.F. Zimmermann (Hrsg.), Soziale Sicherung und Arbeitsmarkt, Empirische Analyse und Reformansätze, Nomos, BadenBaden.

Coppola, M. und A. Reil-Held (2009): Dynamik der Riester-Rente: Ergebnisse aus SAVE 2003 bis 2008, MEA Diskussionspapier 195-09, Mannheimer Forschungsinstitut Ökonomie und Demographischer Wandel (MEA), Universität Mannheim.

Corsetti, G. (1994): An Endogenous Growth Model of Social Security and the Size of the Informal Sector, Revista Analisis Economico, Vol. 9, No.1, 57-76.

Corsetti, G. und K. Schmidt-Hebbel (1995): Pension Reform and Growth, mimeo, The World Bank.

Deutsches Institut für Altersvorsorge (DIA) (1999): Alterungssicherungssysteme ausgewählter Länder, Köln.

Diamond, P.A. (1965): National Debt in a Neoclassical Growth Model, American Economic Review, Vol. 55, No.5, Part 1, 1126-50.

Diamond, P.A. (1994): Privatization of Social Security: Lessons from Chile, Revista Analisis Economico, Vol. 9, No. 1, 21-33.

Feldstein, M., und A. Samwick (1996): The Transition Path in Privatizing Social Security, NBER Working Paper, Cambridge, Mass.

Fenge, R. (1995): Pareto-Efficiency of the Pay-As-You-Go Pension System with Intergenerational Fairness, Finanzarchiv 52, 357-363.

Fenge, R. (1997): Effizienz der Alterssicherung, Physica-Verlag, Frankfurt.

Gasche, M. (2009): Implizite und explizite Lohnsteuerbelastung in Deutschland, Jahrbuch für Wirtschaftswissenschaften, 60. Jg., 138-167.

Gasche, M. (2008): Renditevergleich zwischen Kapitaldeckungssystem und Umlagesystem, Allianz Dresdner Economic Resarch Working Paper Nr. 115, Frankfurt.

Holzmann, R. (1997): Pension Reform, Financial Market Development and Endogenous Growth: Preliminary Evidence from Chile, IMF Staff Papers, Vol. 44, No. 2, 149-178.

Homburg, S. (1988): Theorie der Alterssicherung, Springer-Verlag, Berlin [u.a].

Homburg, S. (1990): The Efficiency of Unfunded Pension Schemes, Journal of Institutional and Theoretical Economics, Vol. 146, No. 4, 640-647. 
Meinhardt, V., Rietzler, K. und R. Zwiener (2009): Konjunktur und Rentenversicherung gegenseitige Abhängigkeiten und mögliche Veränderungen durch diskretionäre Maßnahmen, IMK-Studies 3/2009.

Kotlikoff, L.J. (1996): Simulating the Privatization of Social Security in General Equilibrium, in: M. Feldstein, Privatizing Social Security, NBER Working Paper, Cambridge, Mass.

Levine, R., and S. Zervos (1998): Stock Markets, Banks, and Economic Growth, American Economic Review, Vol. 88, No. 3, 537-558.

Logeay, C., Meinhardt, V., Rietzler, K. und R. Zwiener (2009): Gesamtwirtschaftliche Folgen des kapitalgedeckten Rentensystems - Zwischen Illusion und Wirklichkeit, IMKReport Nr. 43, November 2009.

Lueg, T., Ruprecht W. und M. Wolgast (2003) Altersvorsorge und demographischer Wandel: Kein Vorteil für das Kapitaldeckungsverfahren?, GDV Volkswirtschaft - Themen und Analysen, Nummer 1, Berlin.

OECD (2009): Pensions at a Glance, OECD, Paris.

Pestieau, P. (1994): Social Protection and Private Insurance: Reassessing the Public Versus Private Sector in Insurance, The Geneva Papers on Risk and Insurance Theory, Vol. 19, No. 2, 81-92.

Pestieau, P. und U. Possen (2000): Investing Social Security in the Equity Market: Does it Make a Difference?, National Tax Journal, Vol. 53, No. 1, 41-57.

Raffelhüschen, B. (1993): Funding social security through pareto-optimal conversion policies, in: B. Felderer (Hrsg.): Public pension economics, Wien, 105-131.

Rajan, R.G. und L. Zingales (1998): Financial Dependence and Growth, American Economic Review, Vol. 88, No. 3, 559-586.

Rios-Rull, J.-V. (2001): Population changes and capital accumulation: The aging of the baby boom, Advances in Macroeconomics, Vol. 1, Issue 1, Article 7.

Schmidt-Hebbel, K. (1998): Chile's Takeoff: Facts, Challenges, Lessons, in: Economic Development, Institute of the World Bank, Washington D.C.

Schnabel, R. (1998a): Intergenerational Distribution and Pension Reform in Germany, Working paper, University of Mannheim.

Schnabel, R. (1998b): Kapitalmarktrenditen und die Rendite der gesetzlichen Rentenversicherung. Working paper, University of Mannheim.

Sinn, H.-W. (2000): Why a Funded Pension System is Useful and Why It Is Not Useful, International Tax and Public Finance, Vol. 7, Numbers 4-5, 383-410.

Solow, R.M. (1956): A Contribution to the Theory of Economic Growth, Quarterly Journal of Economics, Vol. 70, No. 1, 65-94.

Wissenschaftlicher Beirat beim Bundesministerium für Wirtschaft (2009): Akzeptanz der Sozialen Marktwirtschaft, Gutachten vom Oktober 2009, Berlin.

World Bank (1994): Averting the Old Age Crisis, Oxford University Press, Oxford. 


\section{Discussion Paper Series}

Mannheim Research Institute for the Economics of Aging, Universität Mannheim

To order copies, please direct your request to the author of the title in question.

\begin{tabular}{|c|c|c|c|}
\hline Nr. & Autoren & Titel & Jahr \\
\hline $185-09$ & $\begin{array}{l}\text { Francesco Cinnirella, } \\
\text { Joachim Winter }\end{array}$ & $\begin{array}{l}\text { Size Matters! Body Height and Labor Market } \\
\text { Discrimination: A Cross-European Analysis }\end{array}$ & 09 \\
\hline $186-09$ & $\begin{array}{l}\text { Hendrik Jürges, } \\
\text { Steffen Reinhold, } \\
\text { Martin Salm }\end{array}$ & $\begin{array}{l}\text { Does Schooling Affect Health Behavior? } \\
\text { Evidence from Educational Expansion in } \\
\text { Western Germany }\end{array}$ & 09 \\
\hline $187-09$ & Michael Ziegelmeyer & $\begin{array}{l}\text { Das Altersvorsorge-Verhalten von } \\
\text { Selbständigen - eine Analyse auf Basis der } \\
\text { SAVE-Daten }\end{array}$ & 09 \\
\hline $188-09$ & $\begin{array}{l}\text { Beatrice Scheubel, } \\
\text { Daniel Schunk, } \\
\text { Joachim Winter }\end{array}$ & $\begin{array}{l}\text { Don't raise the retirement age! An experiment } \\
\text { on opposition to pension reforms and East- } \\
\text { West differences in Germany }\end{array}$ & 09 \\
\hline $189-09$ & Martin Gasche & $\begin{array}{l}\text { Die sozialversicherungspflichtig Beschäftigten } \\
\text { im deutschen Sozialversicherungssystem: } \\
\text { Eigenschaften, Beitragsleistungen und } \\
\text { Leistungsbezug }\end{array}$ & 09 \\
\hline $190-09$ & Martin Gasche & $\begin{array}{l}\text { Implizite Besteuerung im deutschen } \\
\text { Sozialversicherungssystem }\end{array}$ & 09 \\
\hline 191-09 & $\begin{array}{l}\text { Alexander Ludwig, } \\
\text { Alexander Zimper }\end{array}$ & $\begin{array}{l}\text { Biased Bayesian learning and the risk-free } \\
\text { rate puzzle }\end{array}$ & 09 \\
\hline $192-09$ & $\begin{array}{l}\text { Tabea Bucher- } \\
\text { Koenen }\end{array}$ & $\begin{array}{l}\text { Financial Literacy and Private Old-age } \\
\text { Provision in Germany - Evidence from SAVE } \\
2008 \text { - }\end{array}$ & 09 \\
\hline 193-09 & $\begin{array}{l}\text { Axel Börsch-Supan, } \\
\text { Martin Gasche, } \\
\text { Michael Ziegelmeyer }\end{array}$ & $\begin{array}{l}\text { Auswirkungen der Finanzkrise auf die private } \\
\text { Altersvorsorge }\end{array}$ & 09 \\
\hline 194-09 & Wolfgang Kuhle & The Optimum Structure for Government Debt & 09 \\
\hline $195-09$ & $\begin{array}{l}\text { Michela Coppola, } \\
\text { Anette Reil-Held }\end{array}$ & $\begin{array}{l}\text { Dynamik der Riester-Rente: Ergebnisse aus } \\
\text { SAVE } 2003 \text { bis } 2008\end{array}$ & 09 \\
\hline $196-10$ & $\begin{array}{l}\text { Alexander Ludwig, } \\
\text { Thomas Schelkle, } \\
\text { Edgar Vogel }\end{array}$ & $\begin{array}{l}\text { Demographic Change, Human Capital and } \\
\text { Welfare }\end{array}$ & 10 \\
\hline $197-10$ & $\begin{array}{l}\text { Axel Börsch-Supan } \\
\text { Martin Gasche }\end{array}$ & Zur Sinnhaftigkeit der Riester-Rente & 10 \\
\hline
\end{tabular}

